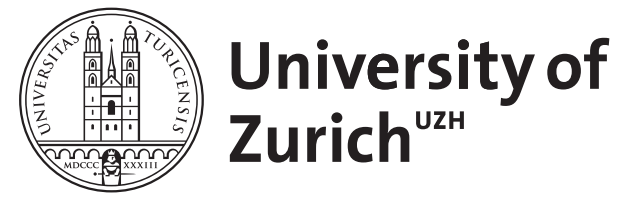

\title{
Wird die Moral von uns geschaffen?
}

Schaber, Peter

DOI: https://doi.org/10.1515/9783110524055-028

Posted at the Zurich Open Repository and Archive, University of Zurich

ZORA URL: https://doi.org/10.5167/uzh-175349

Book Section

Published Version

Originally published at:

Schaber, Peter (2017). Wird die Moral von uns geschaffen? In: Kanzian, Christian; Kletzl, Sebastian; Mitterer, Josef; Neges, Katharina. Realism - Relativism - Constructivism. Berlin: Walter de Gruyter, $365-378$.

DOI: https://doi.org/10.1515/9783110524055-028 


\title{
Peter Schaber
}

\section{Wird die Moral von uns geschaffen?}

\begin{abstract}
It is a widely shared view that moral norms are the outcome of our volitional activity. In this paper it is argued that this view should be rejected. It is shown that none of the proposals to understand moral norms as created by volitional activities are convincing. Moral norms are neither the outcome of demands nor the result of hypothetical agreements. This is, it is argued, what distinguishes moral from other norms such as legal and institutional norms.
\end{abstract}

Keywords: Moral norms, legal norms, institutional norms, constructivism, hypothetical agreements

Verschiedene Autoren vertreten die Ansicht, dass moralische Normen sich nicht einfach in der Welt vorfinden, sondern von Menschen geschaffen werden. So schreibt zum Beispiel Peter Stemmer:

Das moralische Müssen ist [...] ein Müssen, das nicht einfach aus vorgegebenen Umständen resultiert, sondern durch menschliches Handeln erst geschaffen wird [...] Es ist Menschenwerk und insofern ein in diesem Sinne künstliches Müssen. (Stemmer 2000, 118)

Moralische Normen regeln unsere sozialen Beziehungen. Es ist deshalb naheliegend, sie als etwas zu verstehen, das Menschen für genau diese Zwecke schaffen. Viele, die moralische als von Menschen geschaffene Normen verstehen, meinen zudem, dass die einzig und dabei abzulehnende Alternative die Ansicht sei, moralische Normen seien von einem göttlichen Gesetzgeber geschaffen worden (vgl. Stemmer 2000, 6). Wer diese Ansicht für falsch hält, muss, so diese Autoren, moralische Normen für Menschenwerk halten.

Werden moralische Normen allerdings wirklich geschaffen? Es soll nachfolgend dafür argumentiert werden, dass das nicht der Fall ist. Es ist charakteristisch für moralische Normen, dass sie nicht geschaffen wurden und nicht geschaffen werden, weder von Menschen noch von anderen Wesen. Wenn gefragt wird, ob moralische Normen geschaffen werden, dann stehen die richtigen moralischen Normen im Blick und nicht die, welche fälschlicherweise für moralische Normen gehalten werden. Der Auffassung zufolge, die nachfolgend kritisiert werden soll, sind diese Normen das Resultat menschlichen Handelns und zwar des absichtlichen Handelns derjenigen, welche die Normen befolgen sollten. ${ }^{1}$

1 Für diese Auffassung ist folgende These zentral: „(T)hat all normative facts and principles are 
Den unterschiedlichen Interpretationen dieser Doktrin ist die Idee gemeinsam, dass es Normen nur geben kann, wenn Menschen bestimmte Dinge tun und sich in diesem Tun auf die Normen beziehen. Was Menschen dabei tun, ist „truthmaking not truth tracking“. Die moralischen Normen, denen wir folgen sollten, kommen durch absichtliches Handeln von Menschen in die Welt.

Ich werde diese Auffassung nachfolgend zurückweisen, indem deutlich mache, dass keiner der Vorschläge, wie moralische Normen als geschaffene Normen verstanden werden könnten, zu überzeugen vermag. Wir verfügen nicht über die normative Fähigkeit, moralische Normen zu erzeugen. Wie wir sehen werden, sind wir in gewissen Situationen durchaus in der Lage, die moralischen Eigenschaften von Handlungen zu verändern, dies aber bloß auf dem Hintergrund schon bestehender moralischer Normen.

Wenn das, wofür hier argumentiert wird, richtig ist, nämlich dass moralische Normen keine geschaffenen Normen sind, dann heißt das nicht, dass es sie auch ohne Menschen geben würde, sondern bloß, dass sie nicht geschaffen wurden. Nicht bloß die Moral, sondern auch andere Dinge gibt es bloß, weil es Menschen gibt: So gibt es z.B. ein Bewusstsein der Zukunft bloß, weil es Menschen gibt. Keine anderen uns bekannten Wesen besitzen ein solches Bewusstsein. Zukunftsbewusstsein haben Menschen aber nicht geschaffen. Wir haben ein Zukunftsbewusstsein aufgrund bestimmter physischer Eigenschaften. Entsprechend denke ich, dass es moralische Normen bloß gibt, weil es Menschen gibt, die bestimmte Eigenschaften besitzen. Genauso wie das Bewusstsein von Zukunft werden moralische Normen allerdings nicht durch Menschen geschaffen.

\section{Begriffliche Vorbemerkungen}

Moralische Normen würden geschaffen, wenn sie das Resultat intentionalen Handelns wären. Stellen wir uns vor, Menschen würden sich treffen und nach eingehender Diskussion auf die Norm „Man soll Unschuldige nicht töten“ einigen. Sie würden sagen, dass diese Norm von denjenigen, welche sich darauf geeinigt haben, nun auch in Zukunft befolgt werden soll.

Die Beteiligten haben der besagten Norm zugestimmt mit der Absicht, ihr in ihrer Gemeinschaft Geltung zu verschaffen. „Töte keine unschuldigen Menschen“ ist die Norm, die nun gilt. Sie wäre das Resultat intentionalen Handelns vieler Menschen. Sie könnte aber auch das Resultat intentionalen Handelns einzelner

ultimately constructed through the volitional activity of the agents to whom they apply.“ (Wallace 2012, S. 21) 
sein. Wir werden verschiedene Vorschläge, wie moralische Normen durch Menschen geschaffen werden, nachfolgend näher betrachten.

Dabei muss folgendes vorausgeschickt werden: Es gibt verschiedene Arten von Normen: Normen der Klugheit, Höflichkeitsnormen, moralische Normen und andere mehr. Unter Normen verstehe ich das, was wir durch Sollensaussagen zum Ausdruck bringen: „Du sollst mehr Sport treiben“, „Du sollst nicht lügen“. Diese Sollensformen können in einem schwachen und in einem starken Sinn verstanden werden: Im schwachen Sinn sind Gründe gemeint, die dafür sprechen etwas zu tun oder zu unterlassen. Im starken Sinn sind Dinge gemeint, die zu tun wir verpflichtet sind. Nicht alle Sollen sind dabei moralischer Natur. Wenn ich sage „Hans sollte mehr Sport treiben“, dann meine ich, dass es klug von Hans wäre, wenn er mehr Sport treiben würde, und dumm, wenn er dies nicht tun würde, aber nicht verwerflich.

Einige Formen des Sollens sind allerdings moralischer Natur: Ihnen nicht nachzukommen, wäre nicht bloß dumm, sondern verwerflich. Dabei verstehe ich unter moralischen Normen solche, deren Beachtung von allen eingefordert werden kann und deren Verletzung alle berechtigt, den moralischen Missetätern Vorwürfe zu machen. Moralische Normen gehen alle etwas an. Wenn Paul Gerda misshandelt, dann darf nicht bloß Gerda, sondern alle dürfen Paul dazu auffordern, damit umgehend aufzuhören. Und nicht nur Gerda darf ihm Vorwürfe, sondern wir alle dürfen ihm Vorwürfe machen. Dass Paul Gerda misshandelt, ist keine Angelegenheit, die bloß Paul und Gerda betrifft. In gleicher Weise sind die Menschenrechtsverletzungen einer Despotenregimes nichts, was bloß die davon Betroffenen und das Regime etwas angeht. Das heißt: Nicht nur die Bürgerinnen und Bürger des Landes dürfen das Regime dazu auffordern, mit den Menschenrechtsverletzungen aufzuhören. Dazu sind vielmehr alle berechtigt; gleichzeitig dürfen auch alle den Missetätern Vorwürfe machen. Das ist das, was ich nachfolgend unter moralischen Normen verstehen werde. Eine Norm wird als eine moralische Norm verstanden, wenn man sie als eine Norm auffasst, die von allen eingefordert werden kann und die alle zu Vorwürfen gegenüber den Missetätern berechtigt.

\section{Forderungen und Verpflichtungen}

Werden moralische Normen geschaffen? Das ist die Frage: Sind die Normen, denen wir folgen sollten und deren Verletzung alle dazu berechtigt, uns Vorwürfe zu machen das Resultat intentionalen Handelns? Sie könnten, dies der erste Vorschlag, aus Forderungen hervorgehen. Dieser Vorschlag drängt sich auf, weil es Normen gibt, die in der Tat das Resultat von Forderungen sind. Sie bestehen, weil 
Menschen Dinge mit der Absicht tun, sie hervorzubringen. Eine Form, das zu tun, besteht darin, von anderen etwas zu fordern. Gewisse Normen bestehen, weil eine oder mehrere Personen von einer oder mehreren Personen etwas fordern. Aus Forderungen - so der Vorschlag - gehen moralische Normen hervor, sofern die fordernde Person dazu berechtigt ist, Forderungen zu stellen.

Betrachten wir dazu ein Beispiel: Wenn der Rektor einer Universität der Seminarvorsteherin des Philosophischen Seminars aufträgt, die Prüfungsordnung zu ändern, dann sollte sie das tun. Wenn das Straßenverkehrsamt mich anweist, mein Auto vorzuführen, dann sollte ich das tun. Genau dasselbe gilt für die Aufforderung des Steueramts, die Steuererklärung für das Jahr 2015 auszufüllen. Das alles sind Beispiele für Dinge, die wir lediglich tun sollen, weil wir dazu aufgefordert worden sind. Dabei mag es unabhängig davon gute Gründe geben, z. B. die Prüfungsordnung des Philosophischen Seminars zu ändern. Verpflichtet das zu tun wird die Seminarvorsteherin jedoch erst durch die Aufforderung des Rektors.

Nicht nur institutionelle, sondern auch rechtliche Normen werden geschaffen. Sie sind das normative Resultat eines intentionalen Handelns von Menschen, und zwar genau von denen, die autorisiert sind, die entsprechenden rechtlichen und institutionellen Normen zu schaffen. So legt die Universitätsordnung der besagten Universität fest, dass der Rektor die Autorität hat, den einzelnen Instituten vorzuschreiben, wie die Dozentinnen und Dozenten die Studierenden prüfen sollen. Und entsprechend kann allein der Rektor die Seminarvorsteherin anweisen, die Prüfungsordnung auf die von ihm gewünschte Weise zu ändern. Würde eine andere Person diese Anweisung erlassen, würde diese normativ ohne Folgen bleiben.

Gehen auch moralische Normen aus Forderungen hervor? Man könnte fragen: Ist die Seminarvorsteherin dem Rektor gegenüber moralisch verpflichtet? Das könnte schon deshalb naheliegen, weil zumindest einige der institutionellen und rechtlichen Normen zugleich auch moralisch gefordert sind. Das Gebot, andere nicht zu töten, ist etwa nicht nur rechtlich, sondern zugleich auch moralisch geboten. Die konkreten Rechtsgebote, andere nicht zu töten, sind zu einem bestimmten Zeitpunkt vom Gesetzgeber erlassen worden. Damit wurde, so könnte man sagen, ein rechtliches und zugleich ein moralisches Gebot geschaffen.

Der Vorschlag ist allerdings mit folgender Schwierigkeit konfrontiert: Andere nicht zu töten, ist rechtlich wie moralisch geboten. Das moralische Gebot, dies nicht zu tun, bestand allerdings schon bevor das Gesetz erlassen wurde. Durch die Gesetzgebung wurde eine moralische in eine rechtliche Norm verwandelt. Das gilt auch für institutionelle Normen: Wenn Dozierende moralisch verpflichtet sind, die Arbeiten der Studenten fair zu beurteilen, dann wird mit der entsprechenden institutionellen Regelung eine moralische in eine institutionelle Norm transformiert. 
Die Möglichkeiten, wie moralische Normen durch Forderungen geschaffen werden könnten, sind damit nicht erschöpft. Betrachten wir folgendes Beispiel: Hans tritt Gerda auf die Füße (Darwall 2006, 67). Das tut ihr weh. Hans sollte seine Füße wegnehmen. Er ist moralisch verpflichtet, das zu tun. Gerda kann das von Hans fordern („Nimm deine Füße weg“). Dieses Beispiel könnte so gelesen werden, als erschaffe Gerda hier eine Norm. Dies ist jedoch irreführend. Es ist nicht Gerdas Forderung, die Hans verpflichtet, seine Füße wegzunehmen, was sich durch folgende Überlegung deutlich machen lässt: Stellen wir uns vor, Gerda würde es nicht wagen, das zu fordern, weil sie eine sehr schüchterne Person ist. Ungeachtet dessen hätte Hans dennoch die Pflicht, Gerda keine Schmerzen zuzufügen. Und wenn Gerda Hans auffordert, seine Füße wegzunehmen, dann macht sie Hans bloß auf seine bereits bestehende moralische Pflicht aufmerksam und erzeugt Druck, das auch zu tun.

Es könnte allerdings sein, so ließe sich einwenden, dass das auf Pflichten der genannten Art, nicht aber auf alle moralische Pflichten zutrifft. Es könnte moralische Pflichten geben, so könnte man argumentieren, die mit Erschaffen anderer Pflichten entstehen. Nehmen wir noch einmal die Aufforderung des Rektors, die Prüfungsordnung zu ändern. Er verpflichtet die Seminarvorsteherin damit, diese Änderung vorzunehmen. Dazu war sie davor nicht verpflichtet. Könnte es sich dabei zugleich auch um eine moralische Verpflichtung handeln? Würde die Institutsleiterin moralisch falsch handeln, wenn sie sich weigern würde, die Prüfungsordnung zu ändern? Wenn das der Fall wäre und die Pflicht vor der Aufforderung des Rektors noch nicht bestanden hätte, dann wäre es naheliegend, dass sie ebenfalls durch die Forderung des Rektors erschaffen worden wäre. Handelt es sich um eine moralische Norm, die geschaffen wurde?

Die Seminarvorsteherin ist verpflichtet, die Prüfungsordnung zu ändern. Sie ist dem Rektor gegenüber verpflichtet, das zu tun und sonst niemandem. Er ist autorisiert, von ihr die Einhaltung der Norm, die er erzeugt hat, zu fordern. Andere können sie natürlich darauf aufmerksam machen, dass es eine Norm gibt, die sie $\mathrm{zu}$ befolgen hat. Das ist aber nicht dasselbe wie die Einhaltung einer Norm zu fordern. Wer autorisiert ist, dies zu tun, ist nämlich auch autorisiert, den Normadressaten bei Nicht-Einhaltung der Norm zur Rechenschaft zu ziehen. Das kann in dem beschriebenen Fall bloß der Rektor tun. Würde die Seminarvorsteherin der Forderung des Rektors nicht nachkommen, wäre er dazu berechtigt, von ihr zu fordern, sich zu rechtfertigen. Würde sie das nicht tun oder nicht in einer Weise, die er als zufriedenstellend ansehen würde, könnte er ihr auch berechtigterweise Vorwürfe machen. Würde eine Drittperson sie auffordern, sich zu rechtfertigen, könnte sie mit Recht erwidern: „Das geht sie nichts an, kümmern sie sich um ihre eigenen Angelegenheiten“.

Daran wird deutlich, dass die Norm, um die es hier geht, keine moralische ist. 


\section{Hypothetische Einigung 1}

Meine bisherigen Überlegungen zeigen nicht, dass moralische Normen nicht geschaffen wurden. Sie zeigen bloß, dass moralische Normen nicht durch Forderungen geschaffen werden. Sie könnten sich aber den Forderungen vorausliegenden intentionalen Handlungen von Menschen verdanken. Man könnte argumentieren, dass sich Menschen vorgängig darauf geeinigt haben, dass wir einander z. B. keine Schmerzen zufügen dürfen. Diese Norm wird im Hans/Gerda Beispiel wirksam. Mit Einigung können dabei unterschiedliche Dinge gemeint sein. Die Einigung kann eine faktische sein: Menschen haben sich zu einem identifizierbaren Zeitpunkt auf die Norm, dass wir uns nicht ohne guten Grund Schmerzen zufügen sollen, geeinigt. Die Einigung könnte aber auch in dem Sinne eine faktisch sein, als Hans, Gerda und die Menschen, mit denen sie es in ihrem Leben im Wesentlichen zu tun haben werden, sich darin einig sind, dass man sich nicht ohne guten Grund Schmerzen zufügen sollte. Der zu diskutierende Vorschlag lautet: Eine moralische Norm besteht, weil wir dieser Norm zustimmen, wenn sie gefragt würden. Moralische Normen sind das Resultat solcher hypothetischer Einigungen unter nicht idealisierten Bedingungen. Ob eine Einigung über diese Norm zu einem bestimmten Zeitpunkt stattgefunden hat, ist fraglich, aber es ist wohl so, dass die meisten Menschen, mit denen wir es in unserem Leben zu tun haben werden, sich darin einig sind, dass anderen nicht grundlos Schmerzen zugefügt werden sollten.

Ist die besagte Norm also das Resultat einer derartigen Einigung? So wie die Seminarvorsteherin die Prüfungsordnung ändern sollte, weil der Rektor sie dazu aufgefordert hat, würde dann gelten: Hans ist verpflichtet, seine Füße wegzunehmen, weil wir uns einig sind, dass man das tun sollte (und entsprechend fordern würden, wenn wir gefragt würden). Man könnte dann sagen: Die Norm gilt, weil wir unsere Zustimmung zu dieser Norm geben und dies von Hans fordern würden. Bei dieser Idee von Einigung geht es um eine hypothetische Einigung, die im Unterschied zu derjenigen, die wir später noch genauer betrachten werden, unter nicht-idealisierten Bedingungen zustande kommt.

Dieser Vorschlag wirft einige Fragen auf. Was ist der Fall, wenn nicht alle zustimmen würden? Gilt die Norm dann immer noch? Wie viele müssten der Norm ihre Zustimmung geben? Die Mehrheit der Menschen, mit denen wir es zu tun haben werden? Oder reicht eine relativ große Minderheit? Oder müssten alle dieser Norm zustimmen? Unabhängig davon, dass nicht klar ist, ob das wirklich alle tun würden, stellt sich auch die Frage, wieso es denn eigentlich alle sein müssten?

Es ist unklar, welche faktische Einigung eine Norm hervorbringen würde. Das ist ein Problem dieses Vorschlags. Ein anderes ist dies: Wenn faktische Einigungen 
Normen hervorbringen würden, dann müssten wir mit unplausiblen Resultaten rechnen. Nehmen wir an, Hans und Gerda lebten in einer stark männerdominierten Gesellschaft, in der die auch von den meisten Frauen geteilte Meinung vorherrscht, auf Schmerzen von Frauen müsse keine Rücksicht genommen werden. Hans müsste in dieser Welt seine Füße nicht von denen Gerdas wegnehmen, da die Norm, anderen nicht grundlos Schmerzen zuzufügen, bloß für Männer gelten würde. Das werden viele für wenig plausibel halten und entsprechend auch nicht bereit sein, diese Norm auf Männer einzuschränken. Wären hypothetische Einigungen unter nicht idealisierten Bedingungen ausschlaggebend, müssten solche Normen als moralische Normen anerkannt werden.

\section{Hypothetische Einigung 2}

Wenden wir uns dem Vorschlag zu, den die meisten Vertreter der Idee, dass moralische Normen geschaffen würden, für richtig halten. Danach gehen moralische Normen aus einer hypothetischen Einigung unter idealisierten Bedingungen hervor. Dieser Vorschlag liegt faktisch in unterschiedlichen Varianten vor und wird von unterschiedlichen Autoren vertreten. ${ }^{2}$ Die Bedingungen, die dabei im Blick stehen, sind solche, die sich von den Bedingungen unterscheiden, unter denen wir normalerweise über moralische Normen nachdenken und zum Schluss kommen, bestimmte Normen für richtig und andere für falsch zu halten. Die hypothetische Einigung ist eine, die Menschen unter idealisierten Bedingungen erzielen würden. Und die richtigen moralischen Normen sind solche, auf die wir uns unter diesen Bedingungen einigen würden. Sie sind richtig, dies die Idee, weil sie das Resultat eines solchen Verfahrens sind. David Copp sieht dies als die Kernidee einer konstruktivistischen Auffassung moralischer Normen: „[A] constructivist theory defines a hypothetical procedure that could in principle be followed, where the outcome of the procedure is a set of standards that the theory holds to be true because they are yielded by the procedure“(Copp 2013,16). Was spricht dafür, sich an Einigungen unter idealisierten Bedingungen zu orientieren? ${ }^{3}$ Der Grund, das zu tun, kann nur darin liegen, dass man sich im Vergleich mit den Einigungen unter nicht idealisierten Bedingungen die besseren Resultate verspricht. So wird man vermuten, dass Menschen sich unter idealisierten Bedingungen z. B. nicht auf sexistische oder rassistische Normen einigen würden. Das ist es, was für solche

2 So einschlägig (Rawls 1971), (Scanlon 1998), (Darwall 2006), (Southwood 2010) und auch (Stemmer 2000).

3 Dazu ausführlich auch (Enoch 2005). 
hypothetischen Einigungen spricht. Würden wir sie uns nicht mit den besseren Resultaten versorgen, wäre nicht klar, wieso wir uns nicht an Einigungen unter nicht idealisierten Bedingungen orientieren sollten.

In welcher Hinsicht sind die einen Resultate jedoch besser als die anderen? Die Antwort kann nur lauten: Im Blick darauf, was wir suchen, wenn wir über moralische Normen nachdenken, nämlich richtige Antworten. Die idealisierten Bedingungen versprechen uns die richtigen Resultate. Wir würden die idealisierten Bedingungen auch nach diesem Gesichtspunkt auswählen: Nehmen wir an, wir hätten zwei unterschiedliche Vorschläge für idealisierte Bedingungen. Für welche würden bzw. sollten wir uns entscheiden? Wir würden uns für diejenigen entscheiden, die uns mit größerer Wahrscheinlichkeit auf den richtigen Pfad und zu Einigungen führen, welche die richtigen moralischen Normen hervorbringen.

Damit wird aber vorausgesetzt, dass die richtigen moralischen Normen den Einigungen vorausliegen. Die idealisierten Bedingungen erlauben es uns, die richtigen moralischen Normen zu finden. Wenn es nicht das wäre, was wir uns von den idealisierten Bedingungen versprechen, wäre unklar, wieso wir uns an Einigungen unter idealisierten Bedingungen orientieren sollten. Der Grund, das zu tun, kann nur der sein, dass ein Nachdenken unter diesen Bedingungen uns auf den richtigen Pfad führt, auf den nämlich, der uns zu den richtigen moralischen Normen führt.

Klar, es geht bei idealisierten Bedingungen darum, Faktoren auszuschalten, die auf die Meinungsbildung in moralischen Fragen keinen Einfluss haben sollten. Angst, Wut, Macht und Eigeninteresse sind Dinge, die in diesem Kontext genannt werden können. Wieso aber sollten solche Faktoren auf unsere Meinungsbildung keinen Einfluss nehmen? Es ist nicht so, dass sie Einigungen verhindern. Das tun sie nicht. Es ist vielmehr so, dass sie zu Einigungen zu führen scheinen, die nicht die Resultate hervorbringen, die wir haben möchten: nämlich die richtigen. So sollten Normen nach Ansicht verschiedener Autoren z. B. nicht bloß im Lichte des Eigeninteresses erwogen werden, weil wir annehmen, dass uns das vom richtigen Pfad wegführt. Nur diejenigen Faktoren sollten für unsere moralische Meinungsbildung maßgebend sein, die uns zu den richtigen moralischen Normen führen. Die Rechtfertigung, sich an idealisierten Bedingungen zu orientieren, liegt allein darin, dass sie das zu tun.

Von Autoren wie Stemmer wird argumentiert, dass nur unter idealisierten Bedingungen die Normen gewählt werden, deren allgemeine Geltung im besten Interesse der Normadressaten wären. Eine rationale Moral kann, so Stemmer, „nur eine interessenfundierte Moral, wobei die Interessen [...] konvergieren müssen [...] (Stemmer 2000, 204). Stemmer bezeichnet das als einen hypothetischen Kontraktualismus, von dem er sagt: 
Er behauptet nicht wirkliche Agreements, sondern imaginiert einen vormoralischen Raum, um zu zeigen, dass es hier für rationale Individuen im Blick auf ihre eigenen Interessen vernünftig wäre, moralkonstituierende Agreements zu vereinbaren und damit eine Moral zu etablieren (Stemmer 2000, 204).

Das idealisierte Verfahren sichert dabei, dass Menschen die Normen wählen, die sie rationalerweise wählen sollten. So Stemmers Vorschlag.

Damit wird aber kein plausibles Modell des Schaffens von Normen verteidigt, weil nach diesem Vorschlag moralische Normen genau besehen gar nicht geschaffen werden. Stemmer geht vielmehr davon aus, dass die richtigen moralischen Normen diejenigen sind, deren allgemeine Geltung und Befolgung im besten Interesse der Normadressaten sind. Und die idealisierten Bedingungen werden nach Maßgabe dieser Idee richtiger moralischer Normen ausgewählt. Letztere gehen aber dann nicht aus intentionalen Akten von Menschen hervor. Welches die richtigen moralischen Normen sind, ist nach diesem Vorschlag vielmehr vorgängig schon bestimmt. Es sind diejenigen Normen, deren Befolgung im besten Interesse der Normadressaten ist. Die idealisierten Bedingungen ermöglichen es den Normadressaten bloß, diese Normen auch zu entdecken.

\section{Versprechen}

Damit sind die Möglichkeiten, wie moralische Normen geschaffen werden könnten, noch nicht erschöpft. Mit bestimmten Handlungen können wir die moralischen Eigenschaften von Situationen verändern. Mit Einwilligungen verwandeln wir unerlaubte in erlaubte Handlungen, mit Versprechen erzeugen wir Pflichten. Deshalb ist folgender Vorschlag naheliegend, der Vorschlag nämlich, dass moralische Normen aus Versprechen hervorgehen. So könnte man sagen, dass Menschen diejenigen moralischen Normen erzeugen, die einzuhalten sie einander versprechen. Und da wir das nicht in realen Akten tun, müsste man ergänzen: diejenigen moralischen Normen, die einzuhalten wir einander versprechen würden, wenn wir danach gefragt würden. So sollten wir andere nicht quälen, demütigen, betrügen et cetera, weil wir ihnen das versprechen würden, würden wir danach gefragt.

Erzeugen Versprechen also in diesem Sinne moralische Normen? Es gibt zwei Gründe, dies zu bestreiten:

a) Wiederum müsste man sich hier auf hypothetische Versprechen unter idealisierten Bedingungen beziehen (was Menschen unter idealisierten Bedingungen 
einander versprechen würden). Und die Probleme, die mit diesem Vorschlag verbunden sind, haben wir oben gesehen.

b) Ein weiteres Problem des Vorschlags besteht darin, dass Versprechen moralische Normen voraussetzen, um normativ bedeutsam sein zu können. Versprechen bringen nämlich nur dann moralische Pflichten hervor, wenn sie sich auf Handlungen beziehen, die moralisch erlaubt sind. Hans verspricht Gerda, ihr beim Umzug zu helfen. Damit verpflichtet er sich ihr gegenüber. Das tut er aber nur unter der Bedingung, dass die fragliche Handlung (Gerda beim Umzug zu helfen) moralisch erlaubt ist. Stellen wir uns vor, er würde ihr versprechen, einen ungeliebten Konkurrenten umzubringen. Er würde sich damit nicht moralisch verpflichten, den Konkurrenten umzubringen, da das, was er verspricht, moralisch verboten ist.

Wer an der Auffassung, dass Versprechen moralische Pflichten erzeugen, festhalten will, müsste sagen, dass Hans durch sein Versprechen eine Pflichtenkollision erzeugt. Er hat nun die Pflicht, den ungeliebten Konkurrenten nicht umzubringen und gleichzeitig die Pflicht, ihn umzubringen. Nun kann man ganz generell bestreiten, dass es Pflichtenkollisionen geben kann. Man kann aber auch im Besonderen bestreiten, dass man Pflichtenkollisionen selbst erzeugen kann. Das aber wäre der Fall, wenn Versprechen in jedem Fall moralische Pflichten erzeugen würden.

Erzeugen Versprechen aber nicht Pflichten? Hans' Versprechen erzeugt in der Tat eine Verpflichtung, aber keine moralische Pflicht. ${ }^{4}$ Wenn er sein Versprechen nicht hält, kann Gerda ihm Vorwürfe machen, er hätte sich ihr gegenüber nicht loyal verhalten. Er hat mit seinem Versprechen aber keine moralische Pflicht erzeugt, die von allen eingefordert werden kann und alle zu Vorwürfen berechtigt, würde er sein Versprechen nicht halten. In normativer Hinsicht verändert sich durch sein Versprechen bloß etwas zwischen ihm und Gerda. Sein normatives Verhältnis zu allen anderen bleibt durch das Versprechen unberührt. Sie werden von ihm nicht berechtigterweise fordern, den ungeliebten Konkurrenten zu töten, sondern vielmehr das gerade nicht zu tun, also sein Versprechen, das er Gerda gegeben hat, nicht $\mathrm{zu}$ halten. Eine moralische Pflicht wird mit unmoralischen Versprechen nicht geschaffen.

Mit Versprechen können wir Dinge, die moralisch erlaubt in Dinge verwandeln, die moralisch geboten sind. Auf diese Weise können moralische Eigenschaften von Situationen verändern. Das gilt allerdings bloß für Handlungen, die

4 Gilbert redet von einem „commitment“, das der Promissar dem Promittenten gegenüber eingeht; vgl. (Gilbert 2011). 
moralisch erlaubt sind, etwas, das vorgängig feststehen muss. Moralische Verbote können nicht durch Versprechen in Gebote verwandelt werden. Zudem ist es so, dass Versprechen auch keine moralischen Pflichten erzeugen, wenn sie sich auf Handlungen beziehen, die auszuführen wir unabhängig von unseren Versprechen verpflichtet sind. Wenn Hans Gerda verspricht, sie nicht zu vergewaltigen, erzeugt er damit keine moralische Pflicht. Dazu war er nämlich schon davor moralisch verpflichtet. Wir haben die normative Fähigkeit, die moralischen Eigenschaften von Situationen zu verändern, nicht aber die normative Fähigkeit, Normen zu erzeugen.

\section{Eine besondere Autorität?}

Aber wie kommen moralische Normen in die Welt? Werden sie wenn nicht von Menschen so doch von einem Wesen, das eine besondere moralische Autorität besitzt, geschaffen? Nein. Sie werden von niemanden geschaffen. Das lässt sich an folgendem verdeutlichen: Wenn moralische Normen von einem Wesen geschaffen würden, wäre die Einhaltung der geschaffenen Normen etwas, das wir dem Normengeber schulden. Er ist berechtigt, uns zur Rechenschaft zu ziehen. Das ist der Fall bei rechtlichen und institutionellen Normen: Wir schulden ihre Einhaltung dem Normengeber: Der Rechtsgemeinschaft im Fall rechtlicher Normen und dem Inhaber normativer Autorität im institutionellen Fall. Wenn man rechtliche und institutionelle Normen verletzt, bringt man dem Normengeber nicht die angemessene Achtung entgegen. Die Seminarvorsteherin schuldet es dem Rektor, die Prüfungsordnung zu ändern. Er hat die Norm geschaffen und er kann entsprechend die Einhaltung seiner Norm einfordern und der Seminarvorsteherin Vorwürfe machen, wenn sie seiner Forderung nicht nachkommt. In gleicher Weise schulden Bürger die Einhaltung der Rechtsnormen der Rechtsgemeinschaft. Und genau das wäre auch der Fall, wenn moralische Normen von einem Wesen mit besonderer Autorität geschaffen würden. Wir würden dem moralischen Gesetzgeber die Einhaltung der moralischen Normen schulden; dieses Wesen allein wäre autorisiert, dies von uns zu fordern und uns im Fall der Verletzung der Norm zur Rechenschaft zu ziehen. Und daran wird deutlich, dass es sich bei den Normen, die durch ein solches Wesen erzeugt würden, um keine moralische Normen handeln würde: Moralische Normen können von allen eingefordert werden und alle sind berechtigt, den moralischen Missetätern Vorwürfe zu machen. Nicht bloß Gerda darf von Hans fordern, seine von ihren Füßen zurückzuziehen. Alle dürfen das von ihm fordern und ihm Vorwürfe machen, wenn er es nicht tut. Die Verletzung moralischer Normen geht uns alle etwas an. 
Es lässt sich fragen, ob es die Achtung, die man dem Normengeber auch in institutionellen und rechtlichen Zusammenhängen schuldet, nicht auch Gegenstand einer moralischen Achtungsnorm ist. Wenn das allerdings der Fall wäre, könnte die Seminarvorsteherin von allen aufgefordert werden, die Prüfungsordnung zu ändern und von allen zur Rechenschaft gezogen werden, wenn sie es nicht tun würde. Das ist aber nicht so. Nur der Rektor ist berechtigt, das von ihr zu fordern. Daran wird deutlich, dass in einem solchen Fall keine moralische Verpflichtung vorliegt.

Doch wie kommen moralische Normen in die Welt, wenn sie nicht von Menschen geschaffen wurden? Moralische Normen setzen bestimmte Dinge voraus: Dass es Wesen gibt, die in der Lage sind, Normen zu erfüllen und Pflichten zu befolgen; Wesen, die Adressaten von Forderungen sein und Tadel und Vorwürfe verstehen können. Moralische Normen setzen auch voraus, dass es Wesen gibt, die bestimmte Anliegen und Interessen haben, die beeinträchtigt und verletzt werden können; Wesen, denen bestimmte Dinge wichtig sind und die in der Lage sind, Ansprüche geltend zu machen. So setzt die moralische Norm, anderen nicht ohne guten Grund Schmerzen zuzufügen, voraus, dass es Wesen gibt, die Schmerzen haben und unter Schmerzen leiden können. Und die Norm, andere nicht zu demütigen, setzt voraus, dass Menschen sich als Wesen verstehen, welche die gleichen Rechte und die gleiche Würde wie alle anderen haben. Wesen, auf die das zutrifft, erzeugen nicht die Moral. Vielmehr ist es so, dass mit ihnen moralische Normen entstehen: Die Norm, anderen nicht ohne guten Grund Schmerzen zuzufügen, entsteht, weil Menschen bestimmte Eigenschaften haben, nicht durch Menschen. Wie der Zusammenhang zwischen diesen Eigenschaften und moralischen Normen genau beschaffen ist, welche Eigenschaften in welcher Weise moralisch relevant sind, ist der Gegenstand einer substantiellen Theorie moralischer Normen, von Theorien, die zu sagen versuchen, welche Eigenschaften als Gründe zum Handeln und im Besonderen welche als Verpflichtungsgründe anzusehen sind. Genau das ist der Gegenstand normativer Theorien der Moral.

\section{Conclusio}

Rechtliche Normen und institutionelle Normen sind das Resultat intentionalen Handelns. Moralische Normen, so habe ich argumentiert, sind das nicht. Das heißt: Es gibt zwar Akte, nämlich Versprechen, die auch moralische Normen schaffen. Sie tun das aber bloß innerhalb einer moralischen Ordnung dessen, was moralisch verboten, erlaubt und geboten ist. Interessanterweise ist die Idee, dass moralische Normen geschaffen werden, verbreitet. Verbreitet ist sie, weil es naheliegend ist, moralische Normen in Analogie zu rechtlichen und institutionellen 
Normen zu sehen. Wenn letztere geschaffen werden, so die Überlegung, dann auch die moralischen. Doch moralische Normen sollten, so viel sollte deutlich geworden sein, nicht nach dem Modell rechtlicher und institutioneller Normen verstanden werden. Moralischen Normen sind nicht eine Art von rechtlichen Normen, sondern Normen eigener Art, die wir nicht schaffen, sondern vorfinden. Von der Idee, sie seien von jemandem geschaffen worden, sollten wir uns verabschieden.

\section{Literaturverzeichnis}

Copp, David (2013): „Is Constructivism an Alternative to Moral Realism?“. In: Bagnoli, Carla

(Hg.): Constructivism in Ethics. Cambridge: Cambridge University Press, 108-132.

Darwall, Stephen (2006): The Second Personal Standpoint. Harvard: Harvard University Press. Enoch, David (2005): „Why Idealize?“. In: Ethics 115, 759-787.

Gilbert, Margaret (2011): „Three Dogmas about Promising“. In: Sheinman, Hanoch (Hg.):

Promises and Agreements. Oxford: Oxford University Press, 80-108.

Rawls, John (1971): A Theory of Justice. Cambridge: Belknap Press.

Scanlon, Thomas (1998): What We Owe to Each Other. Harvard: Harvard University Press.

Southwood, Nicholas (2010): Contractualism and the Foundations of Morality. Oxford: Oxford University Press.

Stemmer, Peter (2000): Handeln zugunsten anderer. Berlin: de Gruyter.

Wallace, R. Jay (2012): „Constructivism about Normativity: Some Pitfalls“. In: Lenman, James; Shemmer, Yonatan (Hg.): Constructivism in Practical Philosophy. Oxford: Oxford University Press, $18-39$. 
Bereitgestellt von | UZH Hauptbibliothek / Zentralbibliothek Zürich Angemeldet Heruntergeladen am | 24.10.19 09:38 